\title{
A novel anatomical titanium plate versus the reconstruction plate for quadrilateral plate fractures of the acetabulum: A propensity-matched cohort study
}

Haiyang Wu

PLA Middle Military Command General Hospital

Ranran Shang

PLA Middle Military command general hospital

Ximing Liu

PLA Middle Military Command General Hospital

Chengjing Song

PLA Middle Military Command General Hospital

\section{Yanzhao Chen}

PLA Middle Military Command General Hospital

xianhua cai ( $\square$ lzgkcxh@163.com)

PLA Middle Military Command General Hospital

\section{Research article}

Keywords: Acetabular fracture; Quadrilateral; Fracture reduction; Fixation; Reconstruction plate; Models, Anatomic

Posted Date: January 20th, 2020

DOI: https://doi.org/10.21203/rs.2.21289/v1

License: (c) (1) This work is licensed under a Creative Commons Attribution 4.0 International License. Read Full License 


\section{Abstract}

Background Because of the peculiar anatomy and complex geometry of this region, surgical treatment of acetabular fractures involving quadrilateral plate is a challenge to orthopaedic surgeons. Many scholars consider that quadrilateral plate exist a absolute "dangerous zone" for screw placement and have designed a variety of buttress plates to control medial displacement of the quadrilateral surface. In order to achieve the safe and effective fixation in quadrilateral plate, we have developed a novel fixation technique using a special shaped reconstruction plate combined with several buttress screws of quadrilateral plate which was called Dynamic Anterior Plate-Screw System for Quadrilateral plate (DAPSQ) since 2005(RP group). And the long-term follow-up results have confirmed the effectiveness and safety of this technique. After 2016, a set of anatomical titanium plate(ATP group) of DAPSQ have been designed and applied for the treatment of quadrilateral plate fractures. The aim of this study was to compare the efficacy of anatomical titanium plate versus the reconstruction plate in the treatment of quadrilateral plate fractures. Methods We led a propensity-matched cohort study of quadrilateral plate fractures, and twenty-two patients were treated with anatomical titanium plate of DAPSQ in the inclusion period(2016-2018), and were matched to 22 cases in our database of RP group(2008-2016). The primary outcome measures were quality of reduction and functional outcome. Intraoperative conditions included surgical time, bleeding, and blood transfusion and the postoperative complications were also compared. Results Of these 22 consecutive patients in ATP group, the mean age was 46.7 years and the most common fracture pattern was a both-column fracture(12cases, $54.5 \%$ ) according to Letournel-Judet classification. The mean follow up period was 23.1 months (range 12-37). There was no significant differences between the two groups with regards the quality of reduction using the Matta radiological criteria and functional outcomes evaluated by the modified Merle d'Aubigné score $(P>0.05)$. Compared with the RP group, the ATP group has a short operation time(245.1 minutes vs. 286.8 minutes, $P=0.020$ ), less intraoperative blood loss $(1136.4 \mathrm{~mL}$ vs. $1777.3 \mathrm{~mL}, \mathrm{P}=0.014)$ and transfusion(780.9 vs. $1256.8 \mathrm{~mL}$, $P=0.035)$. The complication rate was $22.7 \%$ in ATP group, and there was no significantly difference compared with the RP group (54.5\%)( $>>0.05)$. None of the cases in the two groups had quadrilateral screws entering the hip or implant failure. Conclusions The fixation of anatomical titanium plate in quadrilateral plate fractures showed similar result to the reconstruction plate, in terms of quality of reduction and functional outcome. But the utility of anatomical titanium plate had the advantages of shorter operation time, less bleeding and transfusion which is worthy to recommend.

\section{Background}

With the development of industrialization, high-energy injuries caused by traffic or industrial construction accidents are gradually increasing in China, and the incidence of acetabular fractures is also increasing year by year[1]. Acetabulum is an important part of the hip, which is a complex anatomical structure with a lot of vital vessels and nerves surrounded. In order to allow early rehabilitation, improve the functional outcomes and decrease the risk of post-traumatic arthritis, operative reduction and internal fixation has become the "gold standard" for the treatment of displaced acetabular fractures[2,3]. 
Letournel-Judet classification is one of the widely used types of acetabular fractures. According to the acetabular double-column theory, acetabular fractures are divided into 10 categories, including 5 simple fractures and 5 complex fractures[2]. It is worth noting that except for the simple anterior or posterior wall fractures, the other 8 types of acetabular fractures can involve an important anatomical structure called quadrilateral plate. The quadrilateral plate is located in the medial surface of the acetabulum with the deep site and weak bones. When suffering from high-energy trauma, comminuted fracture is common in this area, which always leads to central dislocation of the femoral head. Chang et al.[4] suggested that the reduction of quadrilateral plate fracture played an important role for the surgical results, and more than $80 \%$ of implant failure occurred in this area. Although quadrilateral plate fractures are not formally considered as a separate parameter for the classification of acetabular fractures, it has gradually attracted the attention of orthopaedic surgeons and been considered as an important factor affecting the complexity of surgery[5,6].

Along with the renovation of internal fixation technologies, some scholars have proposed novel fixition strategies including $1 / 3$ tubular plate[7], T-shaped[8] or L-shaped buttress plates[9], and infrapectineal plates[10].These devices are not entirely without any limitations, in most cases, the plate should be placed along the upper or medial edge of the pelvic boundary, and it is extremely difficult to fix the quadrilateral plate directly by screws, and slight improper manipulation can easily cause screws penetrating into the hip. Although many scholars have proposed several methods such as inserting screws under direct vision after hip capsulotomy, increasing fluoroscopic times to determine the location of screws $[11,12]$. The above-mentioned methods will inevitably prolong the operation time and increase the risk of bleeding and infection.

In previous studies, we have presented a novel technique, the Dynamic Anterior Plate-Screw System for Quadrilateral plate(DAPSQ) which was composed of a specially shaped reconstruction plate and several buttress screws of quadrilateral plate(quadrilateral screws) for the treatment of quadrilateral plate fractures since 2005. This fixation method has been patented in China(No. ZL 20132 0106378.0) and the long-term follow-up results have confirmed the effectiveness and safety of it[13]. However, the reconstruction plate of DAPSQ requires special intraoperative shaping according to the patient's anatomical parameters, and repeated shaping may lead to a reduction in the elastic strength of the plate. For the reason, we have improved this internal fixation system, and designed a set of anatomical titanium plate of DAPSQ according to the acetabular anatomic parameters of Chinese.

The primary objective of this study was to compare the efficacy included quality of reduction and functional outcomes of anatomical titanium plate versus the reconstruction plate of DAPSQ in the treatment of quadrilateral plate fractures. Secondary objectives included surgical time, intraoperative bleeding and transfusion, in addition to comparison of postoperative complications between the two groups.

\section{Patients And Methods}




\section{Patients}

After obtaining approval from our institution's ethical committee(ethical review number: 2018024-1), we led a retrospective case-matched cohort study at a level I trauma center of military general hospital between May 2016 to May 2018. Inclusion criteria included all types of acetabular fractures involving quadrilateral plate, treated with anatomical titanium plate of DAPSQ, in patients above 18 years of age and fresh fractures. Exclusion criteria included open or pathologic acetabular fractures, patients with preexisting osteoarthritis of the affected hip. Written informed consents had been obtained from all patients.

Twenty-two consecutive acetabular fracture patients treated with anatomical titanium plate of DAPSQ(ATP group), with a minimum of 1-year of follow-up, were formed group 1. Then, the fractures in group 1 were selected and matched according to age, gender, fracture pattern and surgical approach, to similar cases from our dedicated acetabular plate fracture database which included more than 140 cases treated with the reconstruction plate of DAPSQ from January 2008 to January 2016. This pool of cases was then used to randomly choose individual cases to create a 1:1 ratio, and this matched cohort was group 2(RP group).

\section{Surgical technique}

\section{Preoperative Management}

The initial management of acetabular trauma followed the principles of the Advanced Trauma Life Support (ATLS) and the most important was to keep the stable of vital signs[14]. Subsequently, all patients received routine physical and neurological examination. Radiological assessment included an anterior-posterior(AP) view and Judet views(iliac and obturator oblique views), along with twodimensional computed tomography(CT) and three-dimensional(3D) CT reconstruction. Fracture pattern were classified according to Judet and Letournel classification[2]. Skeletal traction was applied via the femoral condyles or tibial tubercle in all patients. Low molecular weight heparin was routinely used to prevent thrombosis, and the color Doppler ultrasonography of Lower Limb Vessels was performed to exclude deep venous thrombosis. One day before the operation, autologous blood transfusion machine and heterogeneous blood $(\mathbb{1 0 0 0 \mathrm { mL }})$ should be prepared.

\section{Operation procedures}

The corresponding author and another senior orthopedic surgeon performed all surgeries. All the surgeries was performed on a radiolucent table using a standard ilioinguinal approach described by Letournel[15] or combination with Kocher-Langenbeck approach. Through the "middle window" of the ilioinguinal approach, the acetabular anterior column, pelvic boundary and the upper part of quadrilateral plate could be directly exposed or touched. First, the medial dislocation of femoral head was repositioned under manual traction on the leg or the assistant of mechanical lateral traction via a Schanz pin in the proximal femur. Then restore the continuity of the pelvic ring followed a principle of proximal to distal 
and the fracture fragments of ilium wing and anterior column were fixed with screws or plates. Subsequently, push the fracture fragments of acetabular posterior column or quadrilateral plate into its bed with the help of L-shaped spike pusher until a smooth quadrilateral medial surface with no external stepoff was obtained and use a 2 or 3-claw reduction forceps to maintain the reduction.

\section{ATP group}

After the reduction was accomplished in the ATP group, choose a appropriate mode of the anatomical titanium plate of DAPSQ. The anatomical titanium plate was placed on the superior arcuate line, and the ends extended toward the iliac wing and the superior pubic ramus. And according to the placement position, it was divided into three parts: the iliac region, the quadrilateral region and the pubic region(Fig.1). We have preliminary divided the anatomical titanium plate into three models according to the anatomical length of the DAPSQ trajectory and the different length proportion of the three regions measured in the Chinese (Fig.2). After placement, the both ends of the titanium plate were up-warped, and were not firmly against the bone surface. But through special nailing methods, the titanium plate could be fully adhered to the bone surface, and detailed nailing methods have shown in Figure 3. The key surgical procedures were as follows: Fistly, two or more fixition screws on the iliac and pubic region should be first fixed to stabilize the acetabular anterior column. Then with the help of a $4.5 \mathrm{~mm}$ screwdriver, quadrilateral screws were inserted along the pelvic brim and parallel to the surface of quadrilateral plate under direct vision, and only the $1 / 3$ to $1 / 2$ transverse diameter of the quadrilateral screw into the bone of quadrilateral plate to avoid entering the hip. And during the process of nailing, the torsion and elastic recoil of the plate could provide a strong holding force for the quadrilateral screws to block the inward displacement of quadrilateral plate. In addition, make sure that the distal of the quadrilateral screws have exceeded the fracture line by at least $10 \mathrm{~mm}$.

\section{RP group}

Patients in the RP group were used a reconstruction plate. The shaping steps were as follows: Firstly, select a 12 to 16 hole arc-shaped reconstruction plate according to the actual anatomical length. Then the both ends of the plate were reverse twisted and upturned by using a Bender and screwdriver. The torsion angle of the iliac and pubic regions was higher than the radian of the bone surface, so that the both ends of plate were upturned after placed, and the plate in the quadrilateral region slightly incline to the intrapelvic about $15^{\circ}$ (Fig.4). In order to achieve the best effect of screws placement, repeatedly adjustment of the torsion angle and proportion of the three regions was necessary. After well shaped, the nailing methods was similar to the ATP group.

Finally, if the reduction was unsatisfactory through a single ilioinguinal approach or patients complicated with the fracture of acetabular posterior wall, adding the Kocher-Langenbeck approach was a suitable choice. After the nailing process was completed in all patients, repeated fluoroscopy was necessary to confirm the good reduction and no screw entering the hip. After acquiring satisfied fluoroscopy, the operation area should be flushed completely and a drainage tube was placed before wound closed. 


\section{Postoperative Management}

All patients regularly received an intravenous injection of prophylactic antibiotics for 5-7days after surgery. The drainage tube was removed within 3 days ( $24 \mathrm{~h}$ drainage flow $<20 \mathrm{~mL}$ ). Patients started early rehabilitation after awoke from anesthesia and were instructed to non-weight bearing exercises such as passive and active ipsilateral hip flexion or extension motion on the affected limb for 4-6 weeks. Then protected weight-bearing exercises was encouraged till 8-12 weeks, and gradually progress to fullweight bearing at 12 weeks.

\section{Method of assessment and data collection}

Gender, age, fracture pattern, mechanism of injury, fracture side, concomitant injuries, time between injury and surgery, surgical time, intraoperative bleeding, blood transfusion, hospital stay time were all collected. Complications analyzed included deep venous thrombosis, sciatic nerve problem, lateral femoral cutaneous nerve injury, surgical site infections, posttraumatic arthritis, heterotopic ossification, screws penetrating into the hip joint cavity and implant failure.

Quality of reduction was evaluated by 2 senior orthopedic surgeons using the Matta radiological criteria[16] according to immediate postoperative pelvic X-ray(anteroposterior, inlet, outlet, and Judet views) and 3D CT reconstruction. The scores were classified as anatomic $(0-1 \mathrm{~mm})$, imperfect $(2-3 \mathrm{~mm})$, or poor (>3 $\mathrm{mm}$ ) based on the maximal displacement on all views.

Regular outpatient review and follow-up were performed 1, 2, 3, 6, 12 months after surgery and then yearly thereafter. Changes in clinical function, radiographic progress, fracture healing, and complications were recorded. Functional outcomes were assessed using the modified Merle d'Aubigné score[17] at the last follow-up and graded as excellent (18 points), good (15-17 points), fair (13 or 14 points), or poor ( $<13$ points).

\section{Statistical analysis}

Data was coded and analyzed with the statistical package SPSS version 19.0(IBM Corp, Armonk, NY). Continuous variables were expressed as mean \pm standard deviations and categorical variables with absolute frequencies and percentages. Independent-samples $t$ test was used to compare quantitative variables. Chi-square test or Fisher exact test was used to compare categorical variables. While the ranked data were analysed with Mann-whitney U rank sum test. Cohen's Kappa Index was measured to estimate the inter-observer agreement. A value of $P<0.05$ was considered statistically significant. 


\section{Results}

\section{Demographics!}

Twenty-two patients, including 18 males and 4 females with an average age of 46.7 years (range, 23 to 64 years) were treated with anatomical titanium plate of DAPSQ in the inclusion period. Fractures were classified as both columns in 12, anterior column in 2, ACPHT in 4, T-shaped in 2, transverse and transverse with posterior wall in 1, respectively. Randomization resulted in a matching ratio of 1:1 (Table 1).

Patients were evaluated in terms of their demographic variables. There were no significant differences between the groups with regards to gender $(P=0.176)$, age $(P=0.202)$, mechanism of injury $(P=0.646)$, fracture side $(P=0.353)$, concomitant injuries $(P=0.918)$ and mean time between injury and surgery $(P=$ 0.799) (Table 2).

\section{Intraoperative conditions:}

All patients underwent surgery through single ilioinguinal approach(77.3\%) or combined with KocherLangenbeck approach(22.7\%). There were 2 to 4 quadrilateral screws used to control the medial displacement of quadrilateral plate in all patients. For RP group, a 14 or 16 hole reconstruction plate was most commonly used, while type I anatomical plate was most used in ATP group.

The mean surgical time was increased in RP group (mean: 286.8 minutes; range, 185 to 397 minutes) compared with the ATP group(mean: 245.1 minutes; range, 155 to 355 minutes) with a mean difference of 41.7 minutes $(P=0.020)$. Intraoperative mean blood loss and transfusion were significantly lower in the ATP group compared with the RP group(bleeding: 1136.4 vs. $1777.3 \mathrm{~mL}, P=0.014$; transfusion: 780.9 vs. $1256.8 \mathrm{~mL}, P=0.035)$. There was no significant difference in length of postoperative stay in both groups $(P=0.612)$. The mean postoperative stay was 26.8 days in RP group vs 25.7 days in ATP group. Considering the learning curve for the new technique may affect the results, all the cases in the RP group were after 2008, which the technology had been used for more than 3 years.

\section{Quality of reduction:}

The reduction quality of the acetabulum was evaluated by two independent senior orthopedic surgeons according to the Matta radiological criteria. And the degree of agreement between them was $84.1 \%$, with a kappa value of 0.68 , which indicated substantial agreement. 16 cases $(72.7 \%)$ were graded as anatomical reduction in the ATP group, 5 cases(22.7\%) as imperfect, and 1 cases(4.5\%) as poor. In the RP group, 14 patients (63.6\%) showed anatomical reduction, while $6(27.3 \%)$ and $2(9.1 \%)$ patients showed imperfect and poor reduction, respectively. There was no statistically significant difference $(P=0.490)$ 
(Table 2). Postoperative 3D CT reconstruction and X-ray had shown that there was no one quadrilateral screw entering the hip and no one case occurring early fracture displacement or implant failure.

\section{Functional outcome:}

Follow-up was greater than 12 months in all 44 patients. The mean time of follow-up was 23.1 months in ATP group vs 26.1 months in RP group $(P=0.260)$. According to the clinical and radiological evaluation at 12 weeks of follow-up, fracture healing was achieved in all patients. At the last follow-up, the functional outcome in the ATP group according to the modified Merle d'Aubigné score were excellent in 11 cases (50\%), good in 9 cases $(40.9 \%)$, fair in 1 case $(4.5 \%)$ and poor in $1(4.5 \%)$. While the results in RP group were excellent in $9(40.9 \%)$, good in $9(40.9 \%)$ and fair in $4(18.2 \%)$. Although the excellent-to-good rate of ATP group was higher than the RP group( $90.9 \%$ vs $81.8 \%)$, there was no significant difference between the two groups $(P=0.457)$. Detailed data was summarized in Table 3 , and two typical cases were shown in Figs. 5 and 6.

\section{Complications:}

The mean complication rate was $36.4 \%$ in RP group which was higher compared with the ATP group $(18.2 \%)$, but there was no significant difference $(P=0.176)$. In RP group, One patient presented with an iliac vein thrombosis and pulmonary embolism, and a vena cava filter was inserted on the thirth day after operation. Superficial wound infection was observed in 1 patients, and were treated with antibiotics(2 weeks) and superficial wound debridement. Two cases of lateral femoral cutaneous nerve injury had recovered within two months. Four cases had developed into mild post-traumatic arthritis according to the Kellgren-Lawrence osteoarthritis classification system[18]. In addition, lateral femoral cutaneous nerve injury( 1 case) and post-traumatic arthritis(2 cases) were also observed in ATP group, and one case finally required THA at 48 months. Sciatica symptoms developed in 1 patient and recovered after nerve nutrition drugs were applied. None of the patients in two groups had heterotopic ossification or loss of reduction at the last follow-up.

\section{Discussion}

The quadrilateral plate is located in the medial wall of acetabulum, which plays an important role in preventing the femoral head from moving into pelvic cavity. When high-energy injury occurs, the medial displacement of femoral head often results in the fracture of quadrilateral plate, and even central dislocation of femoral head[8-10]. If the hip joint surface can not be restored by good reduction and reliable fixation, it will inevitably result in high complication rates such as joint dysfunction or posttraumatic arthritis[2-4]. It is particularly important to reconstruct the anatomical structure of the acetabulum and femoral head. On the one hand, it is necessary to restore and coincide the rotation center 
of the femoral head and the acetabulum. On the other hand, the fixation of quadrilateral plate needs to overcome the inward and upward force of the femoral head. However, due to the deep site, weake bones and surrounded with many important blood vessels, nerves and tissues, quadrilateral plate fracture has always been a constant challenge to orthopaedic surgeons[5,6].

Currently, a number of fixation methods for the treatment of quadrilateral plate fractures are in use. The most common methods include the reconstruction titanium plate of anterior column combined with lag screw in posterior column[19,20], reconstruction titanium plate of anterior and posterior column[21]. Letournel and Judet[2,15] described the fixation method of quadrilateral plate fractures with long screws inserting along the pelvic brim and through the thin quadrilateral plate. They also commented that this technique was limited if the medial wall of quadrilateral surface was comminuted or accompanied with severe osteoporosis. Although a biomechanical study carried out by Shazar et al.[22] have shown that periarticular long screws could increase the stability of plate fixation, but what can not be ignored is that this method always bears with a high risk of hip penetrating. In most cases, screw holes in the quadrilateral region of the plate are vacant. If insertion is necessary, the direction of the screw should deviate from the joint surface, or a short screw less than $12 \mathrm{~mm}$, otherwise the screw has the risk of penetrating into the hip[10,13]. Abandoning the direct fixation or screws far from the quadrilateral plate means the less stability of the fixation[22]. Although the use of lag screw in posterior column can increase the stability, it requires the high integrity of anterior and posterior column fragments[19,20].

In order to avoid the screws mistakenly entering the hip, the technique of "buttress plating" for quadrilateral plate instead of screws was first described by Mears et al[7]. A one-third tubular plate used in this fashion was bent in an oblique manner and contoured over the pelvic brim to buttress the medial acetabular wall like a spring. However, subsequent studies have found that it was difficult to provide adequate medial buttress for all individuals, and post-operative nursing of turning-body-over could affect the stability of the fixation[9,23]. Then Farid et al.[24] introduced a method of one-third tubular plate combined with cerclage wire for the fixation of quadrilateral plate fractures. Although the fixation effect was increased, it was easy to injure the sciatic nerve and surrounded vessels because the cerclage wire needed to pass through the greater sciatic notch.

Different from the above-mentioned methods, the fixation technique of DAPSQ is a simple and ingenious design based on the concepts of elastic fixation and dynamic compression. Firstly, quadrilateral screws were located on bone surface and fixed under direct vision through the second window of the ilioinguinal approach. This fixation technique of only the $1 / 3$ to $1 / 2$ transverse diameter of the quadrilateral screw into the bone avoided the risk of hip penetrating. Meanwhile, because the quadrilateral screws and the screws fixed at the iliac region and the pubic region of DAPSQ were not on the same plane, the torsion and elastic recoil of the plate could provide a strong holding force for the quadrilateral screws to control the medial displacement of quadrilateral plate. Besides that, there were 2 to 4 quadrilateral screws parallel to the surface of quadrilateral plate which formed a plane like the "bamboo raft". This multi-point elastic fixation is the key to obtain successfully results. Biomechanical Study have shown that the stability of DAPSQ was superior to the fixation of $1 / 3$ tubular plate based on a fracture model of double- 
column$[25,26]$ The main reason may be that the fixation of $1 / 3$ tubular plate only provides a single point support for the quadrilateral plate, and compared with the "bamboo raft" support formed by DAPSQ, the biomechanical stability is obviously at a disadvantage. A biomechanical study performed by Zha etc. [27]also have shown the the reconstruction plate in pelvic brim combined with long screws for quadrilateral plate fracture have more biomechanical stability compared with the quadrilateral spring plates of T-shaped, L-shaped,and H-plate. Finally, although only part of screws enter the bone, this method of direct fixation can accomplish the reduction and fixation of distal fracture fragments in quadrilateral plate. The quality of reduction and functional outcome have confirmed the effectiveness and safety of the DAPSQ.

As the first propensity-matched cohort study that describes the use of anatomical titanium plate and the reconstruction plate of DAPSQ for quadrilateral plate fractures. Our data indicate that the utility of anatomical titanium plate had the advantages of shorter operation time, less bleeding and transfusion. These findings could be explained by the fact that repeated molding of the reconstruction plate was not required in the anatomical titanium plate group, and appropriate model could be individually selected during the operation. At the same time, the process of nailing was more standardized and sequencing, and the corresponding matching apparatus were more well-used and efficient. One could argue that the reconstruction plate can provide more reasonable design to all individuals, so that the plate could be tightly against the bone surface. However, the characteristics of this technique determine that it is not the key element of the internal fixation, but the repeated shaping process may lead to a reduction in the elastic strength of the plate.

In addition, no significant difference was found between the two groups with regards the quality of reduction using the Matta radiological criteria and the functional outcomes evaluated by the modified Merle d'Aubigné score. To our knowledge, there was only one report[28] that used a reconstruction plate placed on the pelvic brim with buttress screws rubbing on the medial surface of the quadrilateral plate to maintain the reduction of the quadrilateral fracture. In their study, 40 cases of quadrilateral plate fracture with mean age 35 years(range, 16-68 years) were enrolled. The anatomical reduction was achieved in $60 \%$ and the rate of excellent-to-good evaluated by the modified Merle d'Aubigné score was achieved in $90 \%$ of the patients. Compared with our study, the difference may come from the difference in fracture patterns and surgical approachs. But, more importantly, although the fixation concept was similar to ours[28], that was to control medial displacement of the quadrilateral surface on the basis of avoiding the risk of hip penetrating. The fixation method was obviously different from ours. Firstly, we have shaped the reconstruction plate with a special way, and the plate was not fully adhered to the bone surface before screws insertion. During the screws placement, the change of torsion angle on the plate could provide quadrilateral screws with the sufficient force of lateral dynamic compression to block the medial displacement of quadrilateral plate. The quadrilateral screws were not only used as buttress screws but also dynamic pressurized buttress screws. Secondly, We have drilled semi-"U" type holes parallel to the surface of quadrilateral plate under direct vision, which not only enable the quadrilateral screws partially fixed the fracture fragments, but also make the screws adhere to the quadrilateral surface tightly. Meanwhile, the quadrilateral screws were placed through a special process, which could make use of the 
elastic retraction of the plate and prevent the screws migration or floating on the bone surface. Finally, we have designed a anatomical titanium plate with the immobilization length and proportion of three regions, which is more convenient to use.

Furthermore, no significant difference was found between the two groups compared with the mean complication rate. As expected, no one had occurred the quadrilateral screws entering the hip. And there was also no one case occurring early fracture displacement or implant failure. In addition, it is worth noting that the fixation method was effective in 6 elderly patients whose age above 60 years. This may suggested that the technique was effective for osteopenic quadrilateral plate fractures, but larger sample sizes were required. In clinical practice, we found that the anatomical titanium plate of DAPSQ has the following advantages compared with the reconstruction plate: Firstly, additional pre-bending procedure of the plate is not needed during the operation, which greatly simplifies the operation process. Secondly, the pre-moulding design of the anatomical titanium plate can provide more stable elastic strength for quadrilateral screws and reduce the loss of elastic strength due to repeated shaping of the reconstruction plate during operation. Finally, the design of the additional screw hole in the quadrilateral region can not only assist the quadrilateral screws nailing, but also can be used to insert the lag screw in the posterior column to improve the fixation effect if necessary. And the process of nailing in the anatomical titanium plate is more standardized and the matching apparatus are more complete, which greatly improves the efficiency of nailing.

There are several limitations in this study. Firstly, this is a propensity-matched cohort study, where the study group originate from a prospective cohort and are matched with the cases from a retrospective database, which may have selection bias. The selection bias can be minimised by matching all the basic data such as age, fracture types and surgical approaches as much as possible but can not be eliminated. One possible bias worth mentioning is that the learning curve for the new technique may affect the results. Finally, the most common fracture type included in this study is double column fracture, which is different from the distribution types of acetabular fractures in clinical practice. This selection bias is mainly caused by the fact that our cases come from a level I trauma center where the most complex acetabular fractures are referred. Therefore, the results of this study need to be interpreted with caution and confirmed with a large sample.

\section{Conclusion}

Quadrilateral screws fixation of DAPSQ is a simple and efficient technique for quadrilateral plate fractures and should be considered in the reconstruction of acetabular fractures. The anatomical titanium plate and the reconstruction plate of DAPSQ have the similar results in terms of quality of reduction and functional outcome. While the anatomical titanium plate had the advantages of shorter operation time, less bleeding and transfusion which is worthy of further promotion and studies.

\section{Abbreviations}


DAPSQ: Dynamic Anterior Plate-Screw System for Quadrilateral plate; AP: anterior-posterior; CT: computed tomography; 3D: three-dimensional; ACPHT: anterior column posterior hemitransverse type; LFCNI: lateral femoral cutaneous nerve injury; THA: total hip arthroplasty

\section{Declarations}

\section{Acknowledgments}

Thanks to Kunming Cheng, for her love and support all through this work.

\section{Funding}

This work was supported by the technological innovation projects of hubei provine (Grant No. 2017ACA099).

\section{Availability of data and materials}

The datasets generated and/or analyzed during the current study are available from the corresponding author by reasonable request.

\section{Authors' contributions}

WHY, SRR, LXM and CXH conceived and designed the study. SRR and SCJ contributed to the data collection. WHY, CYZ, and SRR analyzed the data. SCJ drew the pictures. WHY wrote the manuscript. All authors read and approved the final manuscript and consented to publish this manuscript.

\section{Ethics approval and consent to participate}

This study has been approved by the appropriate ethics committee(the Scientific Board of the Department of Orthopaedic Surgery, PLA Middle Military Command General Hospital ). Details that might disclose the identity of the subjects in the study have been omitted. No benefits in any form have been received or will be received from a commercial party related directly or indirectly to the subject of this article.

\section{Consent for publication}

Not applicable.

\section{Competing interests}

Each author certifies that he has no commercial or any other associations that might pose a conflict of interest in connection with the submitted article.

\section{Author details}


${ }^{1}$ Department of Orthopaedic Surgery, PLA Middle Military Command General Hospital, Wuhan 430070, Hubei, China. ${ }^{2}$ Southern Medical University, Guangzhou 510515, Guangdong, China.

\section{References}

1. Mauffrey C, Hao J, Cuellar D R et al (2014) The epidemiology and injury patterns of acetabular fractures: are the USA and China comparable?. Clin Orthop Relat Res 472(11):3332-3337.

2. Letournel E (1980) Acetabulum fractures: classification and management. Clin Orthop Relat Res 151:81-106.

3. Mardian S, Rau D, Hinz P et al (2017) Acetabular Fractures in an Advanced Age - Current Knowledge and Treatment Options. Acta Chir Orthop Traumatol Cech 84(4):241-246.

4. Chang J K, Gill S S, Zura R D et al (2001) Comparative strength of three methods of fixation of transverse acetabular fractures. Clin Orthop Relat Res 392:433-441.

5. Bastian J D, Tannast M, Siebenrock K A et al (2013) Mid-term results in relation to age and analysis of predictive factors after fixation of acetabular fractures using the modified Stoppa approach. Injury 44(12):1793-1798.

6. Elnahal W A, Abdel K M, Khaled S A et al (2018) Quadrilateral plate fractures of the acetabulum: Proposition for a novel classification system. Injury 49(2):296-301.

7. Mears D C (1999) Surgical treatment of acetabular fractures in elderly patients with osteoporotic bone. J Am Acad Orthop Surg 7(2):128-141.

8. Sen R K, Tripathy S K, Aggarwal S et al (2013) Comminuted quadrilateral plate fracture fixation through the iliofemoral approach. Injury 44(2):266-273.

9. Peter R E (2015) Open reduction and internal fixation of osteoporotic acetabular fractures through the ilio-inguinal approach: use of buttress plates to control medial displacement of the quadrilateral surface. Injury 46 Suppl 1:S2-S7.

10. Qureshi A A, Archdeacon M T, Jenkins M A et al (2004) Infrapectineal plating for acetabular fractures: a technical adjunct to internal fixation. J Orthop Trauma 18(3):175-178.

11. Sebaaly A, Jouffroy P, Emmanuel M P et al (2018) Intraoperative Cone Beam Tomography and Navigation for Displaced Acetabular Fractures: A Comparative Study. J Orthop Trauma 32(12):612616.

12. Keil H, Beisemann N, Schnetzke $M$ et al (2018) Intraoperative assessment of reduction and implant placement in acetabular fractures-limitations of 3D-imaging compared to computed tomography. $J$ Orthop Surg Res 13(1):78.

13. Cai XH, Liu XM, Wang GD et al (2013) Quadrilateral plate fractures of the acetabulum treated by internal fixation with reconstruction titanium plate combined with trans-plate quadrilateral screws via the ilioinguinal approach. Chin J Orthop Trauma 15(2):102-106. (article in Chinese)

14. Frank C J, Zacharias J, Garvin K L (1995) Acetabular fractures. Nebr Med J 80(5):118-123. 
15. Letournel E (1993) The treatment of acetabular fractures through the ilioinguinal approach. Clin Orthop Relat Res 292:62-76.

16. Matta J M (1996) Fractures of the acetabulum: accuracy of reduction and clinical results in patients managed operatively within three weeks after the injury. J Bone Joint Surg Am 78(11):1632-1645.

17. Merle D R (1990) Numerical classification of the function of the hip. 1970. Rev Chir Orthop Reparatrice Appar Mot 76(6):371-374.

18. Kellgren J H, Lawrence J S (1957) Radiological assessment of osteo-arthrosis. Ann Rheum Dis 16(4):494-502.

19. Jung G H, Lee Y, Kim J W et al (2017) Computational analysis of the safe zone for the antegrade lag screw in posterior column fixation with the anterior approach in acetabular fracture: A cadaveric study. Injury 48(3):608-614.

20. Wang H, Utku K, Zhuang Y et al (2017) Post wall fixation by lag screw only in associated both column fractures with posterior wall involvement. Injury 48(7):1510-1517.

21. Kistler B J, Smithson I R, Cooper S A et al (2014) Are quadrilateral surface buttress plates comparable to traditional forms of transverse acetabular fracture fixation?. Clin Orthop Relat Res 472(11):3353-3361.

22. Shazar N, Brumback R J, Novak V P et al (1998) Biomechanical evaluation of transverse acetabular fracture fixation. Clin Orthop Relat Res 352:215-222.

23. Tosounidis T H, Gudipati S, Panteli M et al (2015) The use of buttress plates in the management of acetabular fractures with quadrilateral plate involvement: is it still a valid option?. International Orthopaedics 39(11):2219-2226.

24. Farid Y R (2010) Cerclage wire-plate composite for fixation of quadrilateral plate fractures of the acetabulum: a checkrein and pulley technique. J Orthop Trauma 24(5):323-328.

25. Wu Y D, Cai X H, Liu X M et al (2013) Biomechanical analysis of the acetabular buttress-plate: are complex acetabular fractures in the quadrilateral area stable after treatment with anterior construct plate-1/3 tube buttress plate fixation?. Clinics (Sao Paulo) 68(7):1028-1033.

26. Wu Y D, Cai X H, Zhang M C et al (2013) Finite element analysis of standing position in both-column acetabular fractures fixed by anterior specially-shaped reconstruction plate combined with transplate quadrilateral screws. Chin J of Experimental Surgery 30(10):2175-2177. (article in Chinese)

27. Zha G, Sun J, Dong S et al (2015)A Novel Fixation System for Acetabular Quadrilateral Plate Fracture: A Comparative Biomechanical Study. BioMed Research International 2015:1-8.

28. Karim M A, Abdelazeem A H, Youness M et al (2017) Fixation of quadrilateral plate fractures of the acetabulum using the buttress screw: A novel technique. Injury 48(8):1813-1818.

\section{Tables}

\section{Table 1}


Fracture types and surgical approach in the anatomical titanium plate(ATP) group and reconstruction plate(RP) group

\begin{tabular}{ccc}
\hline & ATP group $(n=22)$ & RP group $(n=22)$ \\
\hline Fracture type $(n, \%)$ & $12(54.5 \%)$ & $12(54.5 \%)$ \\
Both column & $2(9.1 \%)$ & $2(9.1 \%)$ \\
Anterior column & $4(18.2 \%)$ & $4(18.2 \%)$ \\
ACPH & $2(9.1 \%)$ & $2(9.1 \%)$ \\
T type fracture & $1(4.5 \%)$ & $1(4.5 \%)$ \\
Transverse and posterior wall & $1(4.5 \%)$ & $1(4.5 \%)$ \\
Transverse fracture & $17(77.3 \%)$ & $17(77.3 \%)$ \\
Surgical approach( $n, \%)$ & $5(22.7 \%)$ & $5(22.7 \%)$ \\
Ilioinguinal & & \\
Ilioinguinal+Kocher-Langenbeck & &
\end{tabular}

\section{Table 2}

Comparison of the different variables between the ATP and RP groups

\begin{tabular}{|c|c|c|c|}
\hline Variable & ATP group $(n=22)$ & $\operatorname{RP}$ group $(n=22)$ & $P$-value \\
\hline Gender, male (n, \%) & $18(81.8 \%)$ & $14(63.6 \%)$ & 0.176 \\
\hline Age,years (mean \pm SD) & $46.7 \pm 11.6$ & $50.8 \pm 8.9$ & 0.202 \\
\hline Mechanism of injury $(n, \%)$ & & & \\
\hline Fall from height & $11(50 \%)$ & $8(36.4 \%)$ & 0.646 \\
\hline $\begin{array}{l}\text { Traffic accident } \\
\text { Others }\end{array}$ & $9(40.9 \%)$ & $11(50 \%)$ & \\
\hline Fracture side,left (n, \%) & $15(68.2 \%)$ & $12(54.5 \%)$ & 0.353 \\
\hline $\begin{array}{c}\text { Concomitant injuries }(n, \%) \\
\text { Head trauma }\end{array}$ & $5(227 \%)$ & $6(273 \%)$ & 0018 \\
\hline Spine fracture & $1(4.5 \%)$ & $2(9.1 \%)$ & \\
\hline Limb fracture & $9(40.9 \%)$ & $6(27.3 \%)$ & \\
\hline Rib or clavicle fracture & $4(18.2 \%)$ & $5(22.7 \%)$ & \\
\hline Dislocation of hip & $3(13.6 \%)$ & $2(9.1 \%)$ & \\
\hline Others & $3(13.6 \%)$ & $2(9.1 \%)$ & \\
\hline Time to surgery, days (mean \pm SD) & $8.8 \pm 3.3$ & $8.6 \pm 2.6$ & 0.799 \\
\hline Surgical time, min (mean \pm SD) & $245.1 \pm 54.6$ & $286.8 \pm 59.3$ & 0.020 \\
\hline Blood loss, $\mathrm{mL}$ (mean $\pm \overline{\mathrm{S}} \mathrm{D})$ & $1136.4 \pm 686.3$ & $1777.3 \pm 944.6$ & 0.014 \\
\hline Blood transfusion, mL (mean \pm SD) & $780.9 \pm 685.2$ & $1256.8 \pm 763.2$ & 0.035 \\
\hline Hospital stay time, days (mean \pm SD) & $25.7 \pm 6.3$ & $26.8 \pm 7.8$ & 0.612 \\
\hline Quality of reduction(n, \%) & & & \\
\hline Anatomic $(0-1 \mathrm{~mm})$ & $16(72.7 \%)$ & $14(63.6 \%)$ & 0.490 \\
\hline Imperfect (2-3mm) & $5(22.7 \%)$ & $6(27.3 \%)$ & \\
\hline Poor $(3)+3 x)$ & & & \\
\hline
\end{tabular}

Bold entries indicates statistically significant $P$ value.

Table 3

Postoperative outcome measurements

\begin{tabular}{|c|c|c|c|}
\hline & ATP group $(n=22)$ & $\operatorname{RP} \operatorname{group}(n=22)$ & $P$-value \\
\hline $\begin{array}{l}\text { Mean time of follow-up, month (mean } \pm \text { SD) } \\
\text { Modified Merle d'Aubioné score }(n, \%)\end{array}$ & $23.1 \pm 8.9$ & $26.1 \pm 8.8$ & 0.260 \\
\hline Excellent & 11( & $9(40.9 \%)$ & 0.457 \\
\hline Good & $9(40.9 \%)$ & $9(40.9 \%)$ & \\
\hline Fair & $1(4.5 \%)$ & $4(18.2 \%)$ & \\
\hline Poor & $1(4.5 \%)$ & 0 & \\
\hline Rate of excellent-to-good(\%) & $90.9 \%$ & $81.8 \%$ & \\
\hline
\end{tabular}

Table 4

Descriptive data of postoperative complications 


\begin{tabular}{cccc}
\hline & $\begin{array}{c}\text { ATP group } \\
(n=22)\end{array}$ & $\begin{array}{c}\text { RP group } \\
(n=22)\end{array}$ & $P$-value \\
\hline Deep venous thrombosis & 0 & 1 & \\
Sciatic nerve problem & 1 & 0 & \\
Lateral femoral cutaneous nerve injury & 1 & 2 & \\
Superficial wound infection & 0 & 1 & \\
Posttraumatic arthritis & 2 & 4 & \\
THA & 1 & 0 & \\
Incidence of complication $(n, \%)$ & $4(18.2 \%)$ & $8(36.4 \%)$ & 0.176 \\
\hline
\end{tabular}

\section{Figures}




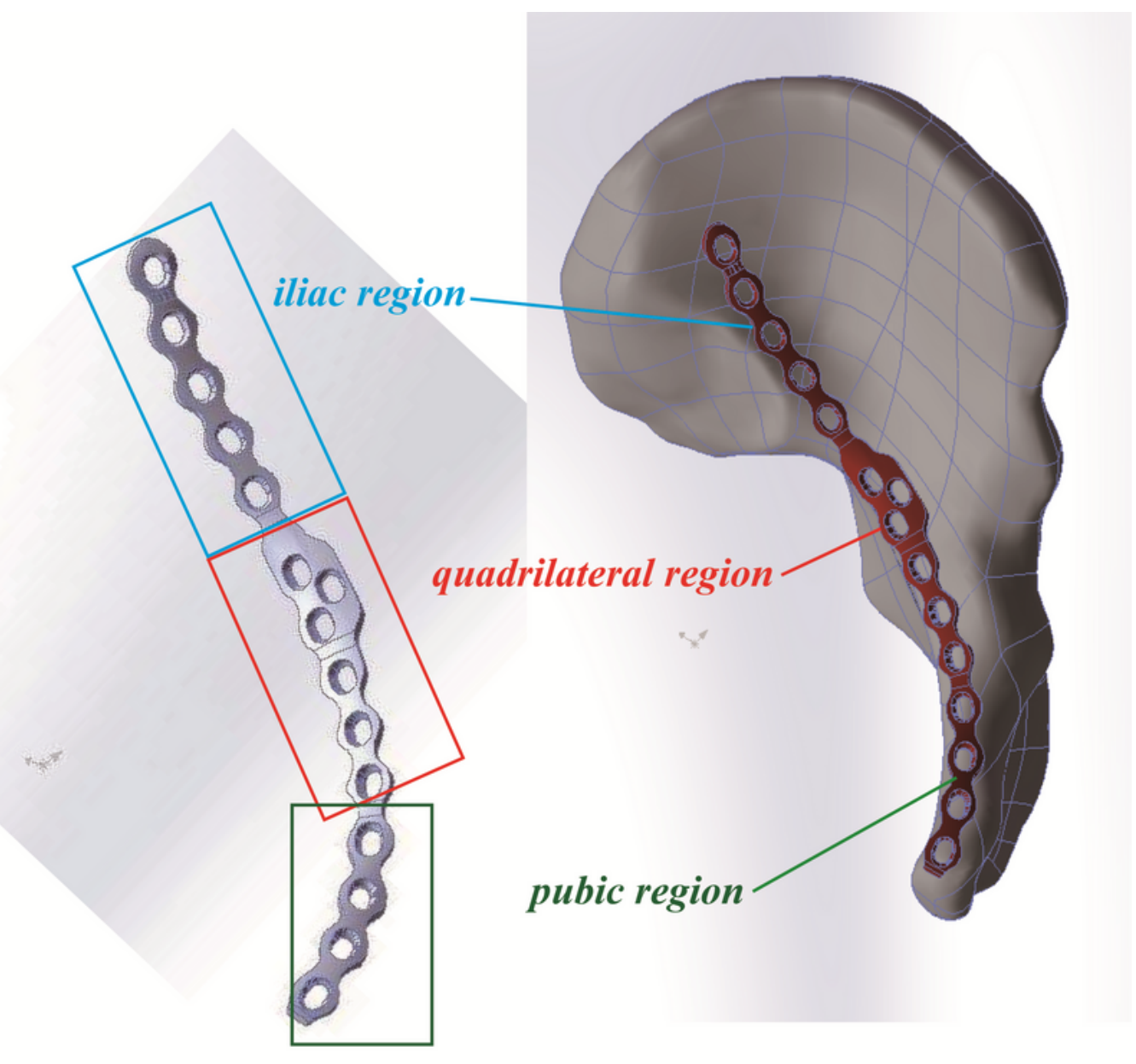

\section{Figure 1}

The structure and components of DAPSQ DAPSQ was placed on the superior arcuate line, and the ends extended toward the iliac wing and the superior pubic ramus, respectively. According to the placement position, it was divided into three parts: the iliac region, the quadrilateral region and the pubic region. And the screws placed in the quadrilateral region were called "quadrilateral screws". 


\section{Figure 2}

Three different models of the anatomical titanium plate According to the anatomical length of the DAPSQ trajectory and the different length proportion of the iliac, pubic and quadrilateral region measured in the Chinese human pelvis, we have divided the anatomical titanium plate of DAPSQ into three models: I, II and III. The appropriate model of titanium plate can be individually selected during the operation.

\section{Figure 3}

The placement sequence of screws The Cadaveric model (The Southern Medical University Anatomy Laboratory, Guangzhou, China) depicted the placement sequence of screws. Choose a appropriate model of titanium plate which was placed along the superior arcuate line. After placement, the both ends of the titanium plate were up-warped (a). The first screw was fixed on the the pubic region(b), and then drill parallel to the surface of quadrilateral plate(c). In this process, attention should be paid to avoid the drill bit entering the bone completely and mistakenly into the hip cavity. After that, insert the first quadrilateral screw along the pelvic brim and parallel to the surface of quadrilateral plate under direct vision, and only the $1 / 3$ to $1 / 2$ transverse diameter of the quadrilateral screw into the bone of quadrilateral plate(d). Then move the plate of the iliac region away from the pelvic rim, so that the first quadrilateral screw can fit snugly against the medial surface of quadrilateral plate冈and insert a screw in the iliac region(e). Subsequently, two fixation screws were inserted into the pubic and the iliac region, respectively, to stabilize the acetabular anterior column(f). Then, a special method of quadrilateral screw placement was adopted. Firstly, a $4.5 \mathrm{~mm}$ screwdriver was inserted into the adjacent screw hole to lift the internal edge of the plate, so that the $1 / 3$ to $1 / 2$ of the screws hole were exposed to the inner edge of the pelvic brim. Then drill and insert the second quadrilateral screw $(\mathrm{g}, \mathrm{h})$. After the placement was completed, the screwdriver was removed, and the plate can naturally bounce back to provide the elastic retraction force for quadrilateral screws to block the inward displacement of quadrilateral plate. The third and fourth quadrilateral screws were inserted in the same way(i). Finally, the residual screw holes at both ends were placed, and the titanium plate was fully adhered to the bone surface(j).

\section{Figure 4}

The shaping method of the reconstruction plate (a) Firstly, the actual anatomical length of the plate placed on the pelvis was measured. (b) Then select a appropriate length of the arc-shaped reconstruction plate. (c) Both ends of the plate were reverse twisted and upturned. And the torsion angle of the iliac and pubic regions was higher than the radian of the bone surface, so that the both ends of plate were upturned after placed. (d) The plate in the quadrilateral region slightly incline to the intrapelvic about $15^{\circ}$. 2

\section{Figure 5}


A typical case showing the use of the reconstruction plate for a displaced acetabular fracture. A 48-yearold man presented with both column fracture of the left acetabulum following a fall from a height. Preoperative AP (a) and 3D CT reconstruction (b) of the pelvis have confirmed the fracture pattern. Fixation was performed 8 days through the ilioinguinal approach using a 12 holes reconstruction plate with 3 quadrilateral screws to control the medial displacement of quadrilateral plate(c). Postoperative CT scanning image(d) and 3D CT reconstruction(e) showing the quadrilateral screws were located on the surface of quadrilateral plate and there was no one quadrilateral screw entering the hip joint cavity. At his two years follow-up visit, the patient was symptom-free. The modified Merle d'Aubigné evaluation was scored as good(f, g).

\section{Figure 6}

The second typical case showing the use of the anatomical titanium plate for a complicated acetabular fracture. A 56-year-old man presented with anterior column and posterior hemitransverse fracture of the left acetabulum following a traffic accident. Preoperative AP (a) and 3D CT reconstruction (b,c,d) of the pelvis have confirmed the fracture pattern. Fixation was performed 9 days through the ilioinguinal approach using the typle I anatomical titanium plate with 4 quadrilateral screws. Postoperative $\mathrm{AP}(\mathrm{e})$ and 3D CT reconstruction $(f, g, h)$ showing Matta's $X$-ray evaluation was scored as excellent. The AP(i) and Judet views(j) after one year showing a good radiological result and no evidence of osteoarthritic change. The modified Merle d'Aubigné evaluation was scored as excellent and the hip function of abduction ( $k$ ) and flexion(I) was satisfied.

\section{Supplementary Files}

This is a list of supplementary files associated with this preprint. Click to download.

- stabilityofquadrilateralscrews.MTS

- Theplacementsequenceofscrews.mp4 